\title{
The effects of food intervention on measures of arterial stiffness and microvascular vasodilation: Systematic Review and meta-analysis
}

\author{
H. M. Cheng ${ }^{1}$, D. Farsi ${ }^{1}$ and J. Lara ${ }^{1}$ \\ ${ }^{1}$ Department of Applied Sciences, Faculty of Health and Life Sciences, University of Northumbria at Newcastle, \\ NE1 8ST, UK.
}

Traditionally, flow mediated dilation (FMD) has been the method of choice when evaluating endothelial function ${ }^{(1)}$. However, assessments of microvascular vasodilation and arterial stiffness are non-invasive methods increasingly used during the past two decades. Both techniques are increasingly being adopted as clinical measures of vascular function. However, the evidence of the impact of dietary and nutritional interventions on these methods has not been systematically reviewed nor meta-analysed. Here, a systematic review and meta-analysis will be reported on the current literature surrounding effects of on nutritional interventions vascular function assessed by pulse wave velocity (PWV), pulse wave analysis (PWA) and laser doppler imaging (LDI).

Three major databases including Medline, Scopus and Web of science were thoroughly searched from inception till January 2019. Intervention trials in adults ( $\geq$ than 18 years), reporting on the effect on markers of vascular function including microvascular vasodilation and arterial stiffness were identified. Microvascular vasodilation assessed with the use of Laser Doppler Imaging (LDI), which measured the response to cutaneous perfusion of the forearm with acetylcholine and SNP.

Meta-analysis was undertaken using review manager software (REVMAN). Random effects models were used to pool data from studies. Effect size was reported as standardised mean differences (SMD) with $95 \%$ confidence intervals. Heterogeneity levels were assessed by the $I^{2}$ test, with values over $50 \%$ representing significant heterogeneity.

Out of 1914 publications identified, 74 fulfilled inclusion criteria. 63 studies were meta-analysed the effect of intervention trials on arterial stiffness. This systematic review and meta-analysis revealed that nutrition intervention significantly improved arterial stiffness, measured by pulse wave analysis (PWA) by SMD $0.17(95 \% \mathrm{CI}-0.25$ to $-0.09, p<0.001)$ and pulse wave velocity (PWV) by SMD $0.15(95 \% \mathrm{CI}-0.23$ to $-0.07, p<0.001)$, among adult subjects $>18$ years of age. The heterogeneity levels were low at $8 \%$ for PWA and $0 \%$ for PWV.

In relation to microvascular vasodilation, seven studies were identified. Meta-analysis was not carried out. Only two studies showed significant improvement in endothelium independent microcirculation vasodilation and one study reported a significant improvement in endothelium dependent microcirculation vasodilation. However, the other five studies, using polyphenol supplementation, did not observe any significant improvement on these outcomes.

In conclusion, this review found that nutritional interventions could improve PWV and PWA; however, the magnitude of these results might be of little clinical relevance. This systematic review also revealed that currently, a limited number of studies using LDI show signs of improvement from nutritional intervention. More research is necessary to evaluate and identify interventions that are effective in improving outcomes of microvascular vasodilation and arterial stiffness.

1. Greyling A, van Mil AC, Zock PL et al. (2016) Atherosclerosis 248, 196-202. 